\title{
Functional MRI Study of Gender Effects in Brain Activations During Verbal Working Memory Task
}

\author{
Z. TÜDÖS ${ }^{1}$, P. HOK ${ }^{2}$, P. HLUŠTÍK ${ }^{2}$, A. GRAMBAL ${ }^{3}$ \\ ${ }^{1}$ Department of Radiology, Faculty of Medicine and Dentistry, Palacky University and University \\ Hospital, Olomouc, Czech Republic, ${ }^{2}$ Department of Neurology, Faculty of Medicine and Dentistry, \\ Palacky University and University Hospital, Olomouc, Czech Republic, ${ }^{3}$ Department of Psychiatry, \\ Faculty of Medicine and Dentistry, Palacky University and University Hospital, Olomouc, Czech \\ Republic
}

Received August 9, 2017

Accepted April 25, 2018

On-line July 25, 2018

\section{Summary}

Neuroimaging methods have been used to study differences of brain function between males and females. Differences in working memory have been also investigated, but results of such studies are mixed with respect to behavioral data, reaction times and activated brain areas. We tried to analyze functional MRI data acquired during the working memory task and search for differences of brain activation between genders. 20 healthy righthanded volunteers (10 males and 10 females) participated in the study. All of them were university students or fresh graduates. Subjects underwent block designed verbal working memory task (Item Recognition Task) inside the MRI scanner. Standard singlesubject pre-processing and group fMRI analyses were performed using the FEAT software from FSL library. In the behavioral data, there was no statistically significant difference in the number of correct responses during the task. The task activated similar bilateral regions of frontal, parietal, temporal and occipital lobes, basal ganglia, the brainstem and in the cerebellum, which corresponds to the previous verbal working memory neuroimaging research. In direct comparison, there was no statistically significant difference in brain activation between small samples of male and female young healthy volunteers.

\section{Key words}

fMRI - Verbal working memory - Brain mapping • Gender differences • Sex differences

\section{Corresponding author}

Z. Tüdös, Department of Radiology, University Hospital, I. P. Pavlova 6, 77900 Olomouc, Czech Republic. Fax: +420 588 442 508. E-mail: zbynek.tudos@seznam.cz

Research of differences in higher cognitive functions between men and women using imaging methods is a dynamically evolving area. Frequently searched features include working memory. Although there are several neuroimaging studies on the subject, the results are not consistent. Some of the studies report differences in behavioral data (Speck et al. 2000, Zilles et al. 2016), some differences in laterality of activations or different activation areas (Bell et al. 2006, Goldstein et al. 2005, Speck et al. 2000, Zilles et al. 2016), and some papers have not shown significant differences in either aspect (Haut and Barch 2006, Schmidt et al. 2009). In order to contribute to this topic, we decided to re-analyze previously published data and to directly compare activations in males and females during the verbal memory task using functional MRI (Tüdös et al. 2011).

Twenty volunteers (ten women, ten men with a mean age of $22.5 \pm 1.7$, resp. $24.1 \pm 2.9$ years, $p=0.38$, Mann-Whitney U test) participated in this study. All participants were university students or recent graduates, all were right-handed. Local ethical committee approved research on healthy volunteers and all subjects participated after providing written informed consent. 
Modified item-recognition task in block-design was used to engage the verbal working memory (Smith and Jonides 1998). In our setting, we used rear projection and a mirror mounted on the top of head coil to first display a slide with four target words; participants had to remember the words and their position on the screen. The target words were displayed for $4 \mathrm{~s}$, then there was 7-second blank delay interval followed by single probe word in one of four possible positions displayed for $3 \mathrm{~s}$. Subject had to respond, whether the probe word corresponded to one of the target words and whether it had the same position. A control condition with the same timing was used to subtract visual processing and to keep subject aware. The control task had the same timing but instead of target words we projected ordinal numbers in four positions ("first", "second", "third", "fourth"), which subjects did not have to remember and the probe word ("first" or "second") was always correct. Each single trial lasted $15 \mathrm{~s}$. Two memory trials alternated with two control trials, thus providing 30 -seconds timing of block design. Six such blocks were one 6-minutes functional run, subjects underwent 2 such runs.

MRI data were acquired on 1.5-Tesla scanner (Siemens Avanto, Erlangen, Germany) with a standard head coil. The subject's head was immobilized with cushions to assure maximum comfort and minimize head motion. The MR imaging protocol covered the whole brain with 30 axial slices parallel to the anterior commissure - posterior commissure (AC-PC) line, 5-mm thick, including anatomical $\mathrm{T}_{1}$-weighted images to provide an immediate overlay with functional data, fluid attenuated inversion recovery (FLAIR) images to screen for unsuspected brain lesions and functional $\mathrm{T}_{2}{ }^{*}$-weighted BOLD images during task performance and control state. BOLD images were acquired with gradient echo EPI (TR/TE $=2500 / 41 \mathrm{ms,}$, flip angle $80^{\circ}$, FOV $=220 \mathrm{~mm}$, matrix $64 \times 64$ ) to provide $3.4-\mathrm{mm} \times$ $3.4-\mathrm{mm} \times 5.0-\mathrm{mm}$ resolution. In total, 144 images were acquired per each 6-minute functional run. For better anatomical reference, a high-resolution 3-dimensional scan (magnetization-prepared rapid acquisition gradient echo, MPRAGE) was performed as well.

Numbers of correct responses in males and females was compared using two-tailed t-test. FMRI data processing was carried out using FEAT (6.00 version, FMRI Expert Analysis Tool), part of FSL (FMRIB's Software Library) (Smith et al. 2004). Standard pre-processing was applied: motion correction; slicetiming correction; non-brain tissue removal; spatial smoothing using a Gaussian kernel of FWHM $8.0 \mathrm{~mm}$; and high-pass temporal filtering with sigma $=30.0 \mathrm{~s}$. Time-series statistical analysis was carried out using FMRIB's Improved Linear Model (FILM) with local autocorrelation correction. Registration to standard space images was carried out using FMRIB's Linear Image Registration Tool (FLIRT). Higher-level analyses of average group activation and between-gender differences were carried out using one-sample t-tests and a two-sample t-test as implemented in FMRIB's Local Analysis of Mixed Effects (FLAME) stage 1 and 2 (Woolrich 2008). Z (Gaussianised T/F) statistic images were thresholded using clusters determined by $\mathrm{Z}>3.5$ and a (corrected) cluster significance threshold of $\mathrm{p}=0.05$ (Worsley 2001).

Performance of the task was good in all subjects, mean number of correct responses out of the 24 possible was $22.5 \pm 1.6$ in males and $22.4 \pm 1.3$ in females, this difference was not statistically significant $(\mathrm{p}=0.88)$. Such a result on behavioral level is consistent with most previous studies on neuroimaging of verbal working memory (Bell et al. 2006, Goldstein et al. 2005, Haut and Barch 2006, Schmidt et al. 2009), studies that have found differences in behavioral domain are not consistent, one reporting a better score for males (Zilles et al. 2016), another for females (Speck et al. 2000).

The results of fMRI analyses by gender subgroups revealed similar bilateral regions of activation in the frontal, parietal, temporal and occipital lobes, basal ganglia, the brainstem and in the cerebellum (Fig. 1) which are areas usually activated during verbal working memory tasks (Smith and Jonides 1998, Tüdös et al. 2014, Wager and Smith 2003). Local maxima were localized to the left BA 9 and 46 , in the inferior frontal gyrus (BA 44 and 47), in the left putamen and head of caudate bilaterally. Activations were also clearly visible in the left BA 44, BA 13 and BA 6. All of these areas were activated also in the right hemisphere, but left-sided dominance was obvious. Bilateral symmetrical activations were in the anterior cingulate cortex (BA 32) and the medial portion of the premotor area BA 6 (SMA). Parietal regions of activation involved bilateral intraparietal sulcus and superior parietal lobule (BA 7/40) with a predominance in the left hemisphere. The temporal lobes showed apparent activity in the posterior division of superior temporal gyrus (BA 21/22) and in both hippocampi. The occipital lobes were activated within Brodmann areas 18 and 19. Areas of activation in both cerebellar hemispheres and vermis were large and symmetric. Between-gender analysis revealed no statistically significant differences. 


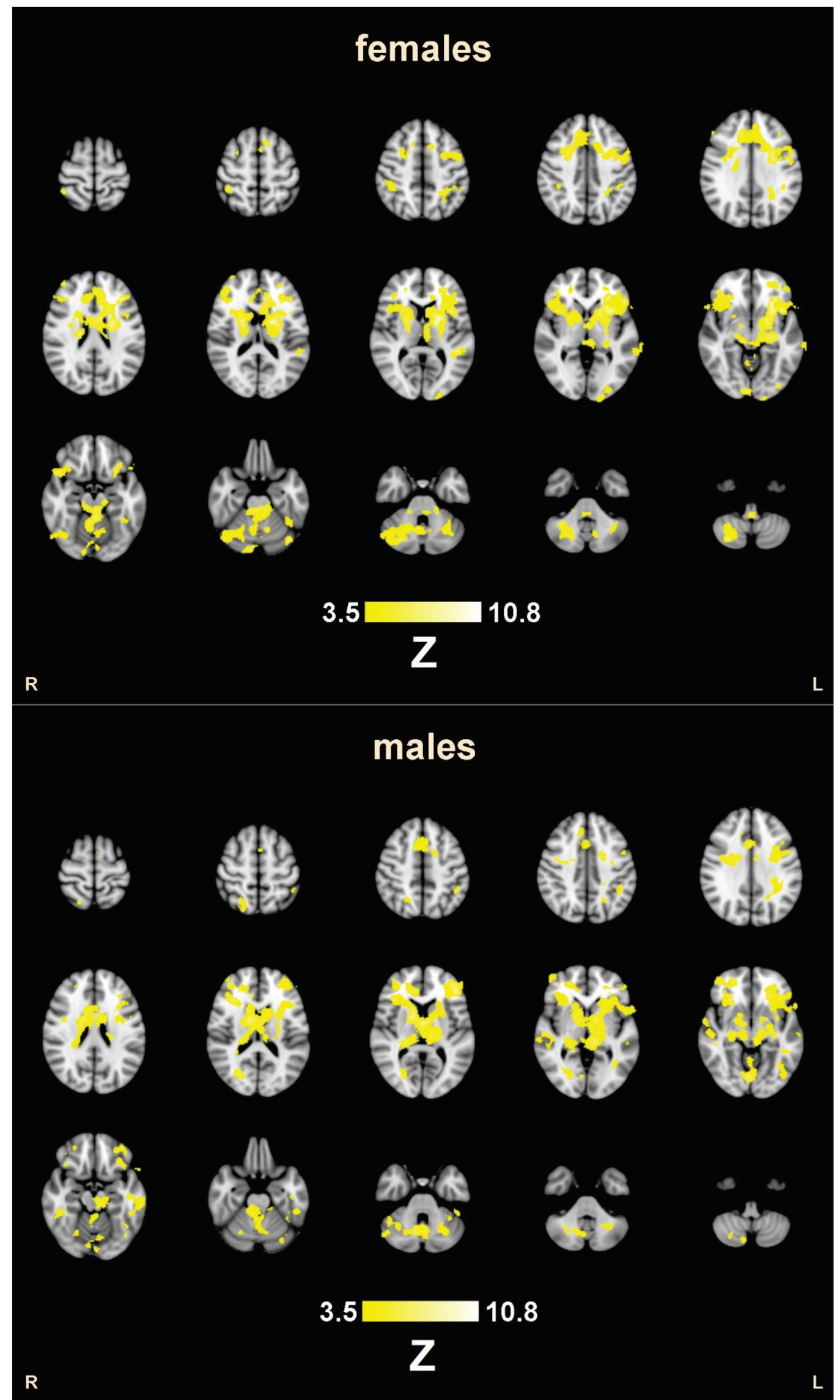

Fig. 1. Group analysis for females (top part) and males (bottom part) reveals similar bilateral regions of activation in the frontal, parietal, temporal and occipital neural networks, basal ganglia, the brainstem and in the cerebellum. Between-gender analysis revealed no statistically significant differences. 
Traditionally, there are assumptions about differences between cognitive functions between males and females. Some previous neuroimaging studies report statistically significant differences between males and females during the verbal working memory task, however, these results are inconsistent - there are reports of increased activation in occipital and parietal areas in 23 males compared to 10 females during the Sternberg item recognition task (Bell et al. 2006), increased bilateral activation in frontal areas in 7 females compared to 7 males during the auditory continuous performance test (similar to the 3-back task) (Goldstein et al. 2005), differences in the laterality index and overall increased activation in 9 males compared to 8 females during the 2-back task (Speck et al. 2000), and finally increased activation in the midbrain and right-sided Broca area in 139 females compared to 92 males (Zilles et al. 2016). This broad range of findings, therefore, does not indicate the existence of convincing gender differences; in general, it can also be concluded that in direct comparison the differences between the genders were significant in smaller or gender unbalanced study groups, and their power is therefore questionable. Published meta-analysis of neuroimaging studies of working memory between the genders also reports the differences; according to their results, females consistently activate more limbic and prefrontal structures and males activate a distributed network including more parietal regions (Hill et al. 2014). Among the drawbacks of the metaanalysis is unbalanced number of subjects (701 male subjects in 44 studies and 200 female subjects in 15 studies). The analysis also pooled results of working memory studies regardless of the nature of the material (e.g. verbal, spatial, etc.), modality of the stimuli (e.g. visual, auditory, cross-modal, other) and difficulty of tasks (e.g. 2-back, 3-back, task timing, etc.), which can lead to misleading results.

Contrary to the above-mentioned inter-gender reports, in our group, there was no statistically significant difference in brain tissue activation between males and females. Schmidt et al. (2009) have also come to the same result using 1-, 2- and 3-back task in 25 males and 25 females. Similarly, another team failed to replicate the statistically significant differences in activation between the genders in two different study groups (the first with 49 subjects and the second with 61 subjects) using verbal 2-back task (Haut and Barch 2006). Our negative result can be further supported by a study that did not show any significant difference in behavioral performance between men and women in both the verbal and spatial working memory task (Kaufman 2007). In a recently published study, gender differences in behavioral data were observed only at very high loads of working memory tasks, i.e. with increasing number of objects to maintain (Reed et al. 2017). In this perspective, working memory load in our task with four objects to remember in each trial was relatively low, which should also be considered when interpreting our negative result.

Our work has several potential limitations. The main limiting factor of our study is the low number of subjects, which reduces the statistical power to detect significant differences. Nevertheless, even the above cited previous studies with larger sample sizes (Haut and Barch 2006, Schmidt et al. 2009) either did not detect any reliable gender effects, or reported them only at an uncorrected statistical threshold (Zilles et al. 2016).

Second, we acknowledge that the current standard for functional neuroimaging studies is the $3 \mathrm{~T}$ MRI scanner, whereas the $1.5 \mathrm{~T}$ field strength used in our study provides a lower spatial and temporal resolution and lower signal-to-noise ratio compared to $3 \mathrm{~T}$ field. To compensate for this, we acquired two functional runs per subject.

Finally, the recruited subjects had a narrow range of age and uniform education level, so our results cannot be generalized to the general population.

In conclusion, our data obtained on a small sample of young healthy volunteers does not indicate a significantly different brain function in the verbal working memory task between the genders. Whereas the brain networks implementing other cognitive functions may be different in males and females, verbal working memory seems to be localized similarly in both genders.

\section{Conflict of Interest}

There is no conflict of interest.

\section{Acknowledgements}

Supported by Palacky University grant IGA_LF_2018_002 and Ministry of Health, Czech Republic - conceptual development of research organization (LF UP 61989592).

\section{References}

BELL EC, WILLSON MC, WILMAN AH, DAVE S, SILVERSTONE PH: Males and females differ in brain activation during cognitive tasks. Neuroimage 30: 529-538, 2006. 
GOLDSTEIN JM, POLDRACK R, BREITER HC, MAKRIS N, GOODMAN JM, JERRAM M, ANAGNOSON R, TSUANG MT, SEIDMAN LJ: Sex differences in prefrontal cortical brain activity during fMRI of auditory verbal working memory. Neuropsychology 19: 509-519, 2005.

HAUT KM, BARCH DM: Sex influences on material-sensitive functional lateralization in working and episodic memory: Men and women are not all that different. Neuroimage 32: 411-422, 2006.

HILL AC, LAIRD AR, ROBINSON JL: Gender differences in working memory networks: a BrainMap meta-analysis. Biol Psychol 102: 18-29, 2014.

KAUFMAN SB: Sex differences in mental rotation and spatial visualization ability: Can they be accounted for by differences in working memory capacity? Intelligence 35: 211-223, 2007.

REED JL, GALLAGHER NM, SULLIVAN M, CALLICOTT JH, GREEN AE: Sex differences in verbal working memory performance emerge at very high loads of common neuroimaging tasks. Brain Cogn 113: 56-64, 2017.

SCHMIDT H, JOGIA J, FAST K, CHRISTODOULOU T, HALDANE M, KUMARI V, FRANGOU S: No gender differences in brain activation during the N-back task: an fMRI study in healthy individuals. Hum Brain Mapp 30: 3609-3615, 2009.

SMITH EE, JONIDES J: Neuroimaging analyses of human working memory. Proc Natl Acad Sci U S A 95: 12061-12068, 1998.

SMITH SM, JENKINSON M, WOOLRICH MW, BECKMANN CF, BEHRENS TE, JOHANSEN-BERG H, BANNISTER PR, DE LUCA M, DROBNJAK I, FLITNEY DE, NIAZY RK, SAUNDERS J, VICKERS J, ZHANG Y, DE STEFANO N, BRADY JM, MATTHEWS PM: Advances in functional and structural MR image analysis and implementation as FSL. Neuroimage 23 (Suppl 1): S208-S219, 2004.

SPECK O, ERNST T, BRAUN J, KOCH C, MILLER E, CHANG L: Gender differences in the functional organization of the brain for working memory. Neuroreport 11: 2581-2585, 2000.

TÜDÖS Z, HLUŠTÍK P, HOK P: Verbal working memory investigation using functional MRI. (In Czech) Ceska Radiol 65: 14-18, 2011.

TÜDÖS Z, HOK P, HRDINA L, HLUŠTÍK P: Modality effects in paced serial addition task: Differential responses to auditory and visual stimuli. Neuroscience 272: 10-20, 2014.

WAGER TD, SMITH EE: Neuroimaging studies of working memory. Cogn Affect Behav Neurosci 3: 255-274, 2003.

WOOLRICH M: Robust group analysis using outlier inference. Neuroimage 41: 286-301, 2008.

WORSLEY KJ: Statistical analysis of activation images. In: Functional MRI: An Introduction to Methods. JEZZARD P, MATTHEWS PM, SMITH SM (eds), Oxford University Press, 2001, pp 266-268.

ZILLES D, LEWANDOWSKI M, VIEKER H, HENSELER I, DIEKHOF E, MELCHER T, KEIL M, GRUBER O: Gender differences in verbal and visuospatial working memory performance and networks. Neuropsychobiology 73: 52-63, 2016. 